\title{
Música para Adultos Mayores: Voluntariado Universitario para la Mejora Social
}

\section{Music for Older Adults: University Volunteer Programme for Social Improvement}

\author{
Susana Sarfson ${ }^{1 *}$ \\ $\mathrm{M}^{\mathrm{a}}$ Claudia Albini ${ }^{2}$ \\ ${ }^{1}$ Universidad de Zaragoza. ${ }^{2}$ Universidad Nacional de las Artes
}

\begin{abstract}
Este trabajo fue realizado durante dos años y respondió a una convocatoria pública y competitiva, diseñado por profesoras de la Universidad Nacional de las Artes (Argentina) y de la Universidad de Zaragoza (España). Los objetivos han sido a) ofrecer actividades musicales para personas ancianas $\mathrm{y}$ b) proporcionar a los estudiantes universitarios la posibilidad de participar en acciones concretas de mejora social. Se ha valorado la influencia de este programa en ambos aspectos. En un marco de investigación-acción, se diseñó el programa de actividades entre docentes y estudiantes; el enfoque es cualitativo. Además del objetivo general (mejora de calidad de vida) se desarrollaron objetivos específicos, diferenciados para ambos grupos de sujetos. Las actividades han sido plasmadas por estudiantes de Música de nivel superior (piano, canto, cuerdas). Se han realizado evaluaciones iniciales, en proceso y final. Los estudiantes universitarios han contribuido a la mejora de calidad de vida de los ancianos y han tomado conciencia de su potencialidad para contribuir a la mejora social. Esto ha permitido validar el programa implementado, y a los estudiantes universitarios vincularse con las necesidades de la sociedad y participar en su mejora.
\end{abstract}

Descriptores: Música, Justicia social, Anciano, Voluntario, Educación musical.

This article presents a work carried out in Argentina for two years as the result of a public, competitive call for proposals. It was designed jointly by professors from the Universidad Nacional de las Artes (Argentina) and the Universidad de Zaragoza (Spain). The aims were: a) to offer quality musical activities to older adults, and b) to provide university students with the opportunity of participating in concrete actions on social improvement. The influence of this programme has been assessed on both aspects. Within a research-action framework, a programme of activities was designed jointly by university professors and students; a qualitative approach was chosen. Within a general aim to improve older adults' life quality, some specific differentiated objectives were developed, according to each group. The activities were performed by students from the faculty of Music (piano, singing, strings). Some initial, in-process and final evaluations were carried out. University students have contributed to a substantial improvement of older adults' life quality through musical activities adapted to each aged group (whether the independent, active community or the dependent one). They have also become aware of their potentiality to contribute to social betterness. This programme has been innovative and has promoted both independent and dependent older adults' well-being; it has also allowed university students of Music become engaged in societal needs and actively participate in social enhancement.

Keywords: Music, Social justice, Elderly, Volunteers, Music education.

*Contacto: sarfson@unizar.es

ISSN: 2254-3139

www.rinace.net/riejs/

revistas.uam.es/riejs
Recibido: $\quad 15$ de enero 2017

$1^{\text {a }}$ Evaluación: 13 de febrero 2017

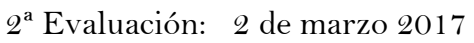

Aceptado: $\quad 13$ de abril 2017 


\section{Introducción}

El envejecimiento de la población es un tema importante en las sociedades actuales. De acuerdo con los estudios demográficos, el perfil de edad de la población de la UE viene experimentando un aumento, y se espera que casi un tercio de los europeos tendrá más de 65 años en 2060 (European Comission, 2012). En la ciudad de Buenos Aires, capital de la Argentina, el panorama es similar: los estudios demográficos indican que la franja etaria predominante es la de los adultos, y solamente existe un predominio de población menor de 20 años en las zonas de asentamientos informales ("villas miseria"). En las partes residenciales de la ciudad, las personas mayores de 65 años van siendo una proporción cada vez mayor dentro de la población general (Di Virgilio, Marcos y Mera, 2015). La preocupación por conservar la autonomía de este sector de la población tiene implicaciones humanas, pero también económicas y sociales. Por otra parte, los estudiantes universitarios de música, debido a la disciplina de estudio técnico-musical, que requiere de muchas horas diarias de dedicación individual en soledad, se pueden ir aislando de la realidad inmediata que los incluye como ciudadanos responsables. Existen estudios que analizan este aspecto en forma específica: cierta falta de comunicación, vida social relativamente pobre y estrés en los pianistas (Ciurana y Cibanal, 2009). Por esto, es importante contribuir también, mediante experiencias del tipo Aprendizaje-Servicio, con la formación personal de los músicos. En 2014, Presidencia de la Nación Argentina, a través del Ministerio de Educación, realizó una convocatoria pública y competitiva para la presentación de Proyectos de voluntariado universitario, fruto de la cual pudo sustanciarse la realización de esta experiencia. Este artículo presenta este programa de voluntariado que fue desarrollado durante dos años en el Departamento de Artes Musicales de la Universidad Nacional de las Artes (Buenos Aires), y describe sus ámbitos de actuación y principales resultados.

\section{Revisión de la literatura}

Las investigaciones más relevantes referidas a la actividad musical (y artística en general) como medio de mejorar la calidad de vida de los adultos mayores y de tratar de conservar las capacidades cognitivas, ponen de manifiesto que la práctica musical puede tener un efecto positivo, que incluye un sentido de bienestar físico y mental, aminoramiento del deterioro cognitivo, sentimiento de placer y disfrute, y alejamiento de sentimientos de tristeza (Lehmberg y Fung, 2010). En Galicia (España) se ha comprobado mediante una investigación amplia, los beneficios de un programa de intervención basado en la psicomotricidad con música en población anciana institucionalizada (Mayán Santos et al., 2001). En Valencia (España), se ha constatado, tras la evaluación de un programa de musicoterapia aplicado a personas con Alzheimer que viven en residencias especializadas, una mejora en la depresión y ansiedad asociadas (De la Rubia, Sancho y Cabanés, 2014). Numerosas investigaciones ponen de manifiesto que la música, ya sea mediante la audición o bien a través de la interpretación, pueden mejorar el rendimiento cognitivo en personas mayores sanas o con patologías neurológicas (Thomson et al., 2005).

Por otra parte, en el contexto donde se ha realizado este proyecto (centros ubicados en la ciudad de Buenos Aires) los ancianos muchas veces ven deteriorado su capital cultural, bien por restricciones económicas o por falta de apoyo social para mantener actividades 
culturales que formaban parte de sus vivencias habituales, lo cual constituye un empobrecimiento de su calidad de vida. Se trata, entonces, de contribuir a evitar o paliar esa creciente exclusión cultural, fortaleciendo, en este caso mediante actividades musicales de calidad, las vivencias de dos grupos de ancianos con necesidades diferenciadas (autoválidos y dependientes).

Además, la sociedad demanda profesionales universitarios socialmente comprometidos (De la Calle, 2004), y es positivo propiciar conexiones entre los estudios académicos (en este proyecto, musicales) y distintos grupos que conforman la ciudadanía. Es necesario la implantación de programas para que los futuros profesionales universitarios desarrollen actitudes y valores, en especial de sensibilidad ante la injusticia global y la exclusión social (Loreto y Matallanes, 2012). Asimismo, se han realizado estudios que evidencian que los proyectos de voluntariado universitario en Argentina facilitan la formación en aspectos que los programas académicos no contemplan, tales como el reconocimiento de la vulnerabilidad, con lo cual los estudiantes pueden incrementar su capital social (Kandel, 2013). Por esto se ha tratado de ofrecer soluciones, variadas, creativas, que pudieran vincular estas cuestiones.

\section{Método y desarrollo de la experiencia: El Programa de voluntariado "En modo mayor"}

A partir de estas cuestiones se diseñó un Programa de Voluntariado destinado a mejorar la calidad de vida de dos grupos concretos de adultos mayores (un grupo dependiente, institucionalizado, y otro grupo de personas mayores, autoválidas) a partir de actividades musicales sistemáticas. Además, el programa debía contribuir a la formación de los estudiantes en cuanto a su integración a la comunidad y al ejercicio de competencias referidas a sensibilización con respecto a las necesidades de los ancianos, y capacidad de emprender mejoras en un contexto social determinado.

Los voluntarios han sido estudiantes de la Licenciatura en Artes Musicales de la Universidad Nacional de las Artes de las especialidades de piano, violín, violoncello, flauta, guitarra y canto, de edades comprendidas entre los 18 y 28 años, todos ellos con experiencia previa en la interpretación en público (solista y de cámara).

Los objetivos concretos fueron los siguientes:

- Incentivar la participación musical de los adultos mayores para fortalecer su autoestima.

- Lograr que los estudiantes universitarios de música se impliquen activamente en la inclusión de personas mayores, ya sea activos física y mentalmente como así también aquellos institucionalizados, a través de actividades musicales de calidad.

- Favorecer una actitud activa de los estudiantes ante la mejora de la comunidad.

- Organizar actividades musicales en hogares para ancianos y en centros de día.

- Propiciar una toma de conciencia en los directivos de la residencia de ancianos dependientes, acerca de los beneficios que la música proporciona, para así incluir actividades musicales permanentes.

- Fomentar la vida social y las relaciones de los mayores mediante la música. 
Para el logro de estos objetivos se articularon las siguientes etapas secuenciadas, que refieren la progresión y preparación de los sucesivos momentos del voluntariado.

- Diseño inicial del programa (profesores universitarios).

- Reunión del equipo con los responsables del Centro de Jubilados y del Hogar de ancianas, para aunar criterios (interés, organización de los encuentros, planificación, horarios, etc.).

- Diseño ajustado del programa: reunión de los estudiantes universitarios voluntarios, docentes y responsables de las instituciones participantes.

- Lectura, estudio y comentario de bibliografía de referencia, especialmente la relacionada con musicoterapia y con métodos para la enseñanza de la música (docentes y estudiantes).

- Selección de material para las sesiones, especialmente en lo referido a repertorio de las audiciones de vivo, ejercicios musicales, canciones, fragmentos de textos literarios, juegos cognitivos, videos, etc.

- Diseño de material pedagógico-didáctico, adecuado para los grupos de ancianos objeto de las experiencias (fichas de lectura de ritmos, partituras con melodías de canciones, materiales auxiliares para psicomotricidad, comentarios para las obras presentadas en audiciones en vivo, selección de instrumentos de pequeña percusión para llevar a las sesiones, etc.).

- En la residencia de ancianas: presentación de los voluntarios a las ancianas dependientes, y participación de los estudiantes en actividades cotidianas para favorecer el mutuo conocimiento y comprender las necesidades de las ancianas.

- Actividades musicales en la Residencia de Ancianas.

- Actividades musicales en los Centros de Día.

- Reuniones de valoración de las experiencias y apoyo a los voluntarios.

- Evaluación final de las actividades: a) Encuestas, b) Entrevistas semiestructuradas a participantes, cuidadoras y familiares, c) Puesta en común con los voluntarios y las profesoras coordinadoras.

Por lo tanto, los contextos donde se ha desarrollado este voluntariado son dos: por una parte, una residencia, donde viven ancianas con dependencia moderada o severa, que cuenta con dos amplios salones cada uno de ellos con un piano. Se trata de un centro de gestión privada, religiosa, ubicado en la ciudad de Buenos Aires, que admite ancianas de diversa condición económica pero que no tienen una red familiar de apoyo suficiente para la etapa de dependencia en la que se encuentran, y que tiende a incrementarse; por otra parte, en centros de día municipales para jubilados autoválidos, que acuden voluntariamente. Por razones básicas de confidencialidad no revelaremos los nombres de los centros participantes ni la identidad de los ancianos.

Las actividades musicales realizadas han sido diferentes en los dos contextos planteados.

En la residencia de ancianas:

- Taller de canto

- Conciertos en vivo (piano, tríos de violín, cello y piano, guitarra, canto y piano). 
- Juegos cognitivos con música, audioperceptiva.

En los centros de día:

- Taller de coro

- Taller de guitarra aplicada al folklore

- Taller de tango en el cine

- Visita al Teatro Colón

- Audiciones musicales en vivo

\section{Resultados}

Todas las sesiones se han registrado mediante diarios, a modo de cuaderno de bitácora, elaborados por los estudiantes de música voluntarios. Asimismo se han completado registros de observación, y se han realizado entrevistas semiestructuradas y encuestas (tanto a los mayores, como a los voluntarios y directivos de la residencia y de los centros de día), para recopilar la información necesaria para valorar el proceso y los resultados parciales, y fundamentalmente para ir ajustando el programa periódicamente.

De la información obtenida se sintetizan los resultados y logros.

\subsection{Voluntarios}

A lo largo del proceso se han realizado reuniones periódicas para apoyar a los estudiantes, tanto en las cuestiones técnico-musicales como, fundamentalmente, en los aspectos emocionales y humanos. Se han propiciado espacios de participación para generar informaciones y reflexión en los participantes, ya que al verbalizar las experiencias y explicar las opiniones se interiorizan conceptos y se mejora la cohesión de los miembros a través de la actividad desarrollada en forma colaborativa. Así, los participantes han manifestado el crecimiento personal que les suponía la experiencia. Ante la cuestión sobre qué les ha aportado esta labor, todos los estudiantes se refieren a una toma de conciencia acerca del valor que tiene el acompañamiento cordial mediante tareas, que puede mejorar el estado de ánimo de las personas mayores, y cómo el hecho de escuchar música en vivo emociona a personas que antes parecían inmersas en su mundo interior, y las hace comunicarse, ya sea verbalmente o, cuando no pueden hablar, mediante las sonrisas, o gestos. Con respecto a quienes desarrollaron juegos cognitivos con elementos musicales, manifiestan que han notado que los ancianos los esperaban cada semana con expectativa, preparados siempre para pasar un buen rato, y que han visto que la actividad lúdica ha contribuido a que se comunicaran más entre ellos. Los estudiantes han manifestado que este voluntariado les ha hecho cambiar su propia percepción ante los ancianos en general, y algunos han comentado un mejor acercamiento a personas mayores de su propia familia o entorno. Desde el punto de vista de la organización de actividades, este voluntariado les ha ayudado a sistematizar y a graduar las propuestas, con una agilización de la capacidad de adaptación al momento concreto, es decir, han desarrollado competencias de gestión. Todos han valorado positivamente también el hecho de intercambiar sus impresiones e ideas en las reuniones de voluntarios y docentes, y en general la experiencia les ha supuesto, a lo largo de los dos años, un desarrollo personal, en especial en un momento de sus vidas donde la mayor parte del tiempo y energías lo están dedicando al estudio de sus respectivos 
instrumentos, con lo cual han podido también conectarse mejor con la realidad circundante. A continuación se reflejan fragmentos extraídos de distintos diarios de los estudiantes voluntarios:

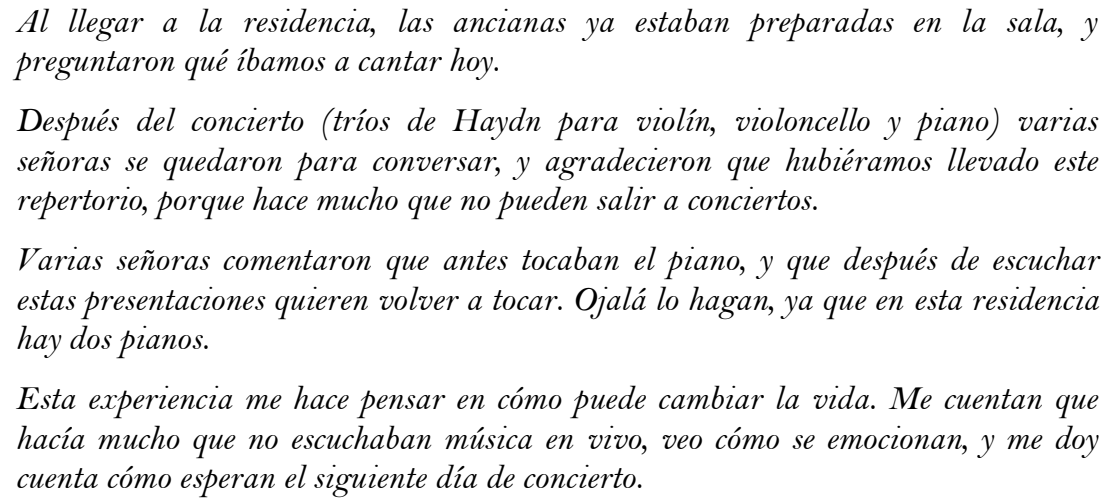

Varias señoras comentaron que antes tocaban el piano, y que después de escuchar estas presentaciones quieren volver a tocar. Ojalá lo hagan, ya que en esta residencia hay dos pianos.

Esta experiencia me hace pensar en cómo puede cambiar la vida. Me cuentan que hacía mucho que no escuchaban música en vivo, veo cómo se emocionan, y me doy cuenta cómo esperan el siguiente día de concierto.

\subsection{Personas mayores}

De acuerdo con las entrevistas semiestructuradas que se realizaron en los distintos momentos (inicial, al finalizar el primer año, y al finalizar el segundo año), los miembros de ambos grupos (residencia y centro de día) han valorado positivamente el programa desarrollado. Sin embargo, las cuestiones que destaca cada grupo difieren: entre los asistentes al centro de día, valoran la posibilidad de aprender (guitarra para acompañar canciones, aspectos de historia de la música, vocabulario musical, canciones), de sentir que ocupan su tiempo en algo constructivo y placentero, así como de relacionarse socialmente, ya que la asistencia a las actividades supone para algunos, continuar la vinculación con sus compañeros fuera del centro. Todos han manifestado que su vida se ha enriquecido. Para las ancianas de la residencia, que tienen dependencia moderada o severa, las que pueden hablar manifiestan la alegría que les supone la llegada de los voluntarios, la emoción de escuchar música, ver los instrumentos, conversar con los músicos; también valoran poder cantar, y los juegos musicales les han parecido no sólo entretenidos sino que les ha permitido ejercitar aspectos del lenguaje verbal (vocabulario, memoria, expresión). Otras ancianas están imposibilitadas de hablar, pero los voluntarios registran las respuestas positivas: sonrisas, apretón de manos, actitud apacible.

A continuación, se transcriben algunos comentarios literales, extraídos de las encuestas realizadas a los sujetos de ambos grupos. Las encuestas realizadas a las ancianas de la residencia se hicieron en forma oral y los voluntarios fueron los encargados de escribir las respuestas.

Centro de día:

La propuesta fue muy adecuada y me gustaría que continuara; me ha permitido tocar la guitarra en clase y practicar en casa.

Excelente el profesor, muy claro y entendible; he aprendido y he disfrutado mucho.

Desearía que la propuesta fuera por más tiempo, no sólo una vez por semana.

Excelente la profesora, muy clara en sus comentarios.

Muy ameno, con material muy rico.

Muy bien elegido el material; trae a la memoria nuestra niñez y juventud. 
Me gustaría repetir el taller, compartimos momentos divertidos y al mismo tiempo aprendimos.

Me emocionó la visita al Teatro Colón.

Excelente predisposición de los voluntarios y de sus profesoras.

Excelentes profesores. Gracias.

Muy profesionales y amenos.

En la residencia de ancianas:

Sería genial tenerlos varias veces por semana.

Muy bonitas todas las canciones que cantamos.

Cantar es un regalo de Dios.

Fuimos muy felices gracias a Uds.

Me hicieron muy feliz con esta música que escuchamos hoy.

Cuando vienen las chicas tengo ganas de salir de mi habitación.

Me siento acompañada y querida.

Espero que pronto tengamos otro concierto.

Con estos ejercicios de canto me siento más derecha así puedo respirar mejor.

Gracias por la compañía, no extraño tanto a mi familia cuando vienen las chicas.

Me divierto y me acuerdo de cuando era chica, cuando mi papá y mi tío tenían un conservatorio en Mendoza.

To canté en el coro de Radio Nacional cuando era jovencita; las chicas me hacen rememorar toda esa época, me emociona.

\subsection{Directivos (residencia y centro de día)}

En ambos casos han valorado muy positivamente este voluntariado, que ha supuesto dinamizar a los centros, y mejorar el bienestar subjetivo de los mayores. En especial en la residencia de ancianas dependientes, han valorado la ruptura de la cotidianeidad mediante actividades enriquecedoras, que han favorecido estados de ánimo positivos en las residentes, y han notado una mejora en cuanto a la capacidad verbal de algunas de ellas inmediatamente después de las sesiones musicales. En ambos casos, los directivos han valorado especialmente la actitud receptiva de los voluntarios, y su predisposición a comunicarse cordialmente con los ancianos, y cómo esto repercutía favorablemente en los grupos.

\section{Discusión y conclusiones}

A lo largo del tiempo de desarrollo de este proyecto (dos años de puesta en práctica, más los meses previos de diseño), se han suscitado diversos temas de reflexión. Así, la necesidad de plantear un voluntariado universitario, que vinculara las necesidades de formación transversal, con la puesta en práctica de las competencias profesionales específicas de estos estudiantes (la interpretación musical y la enseñanza de la música), no estuvo exenta de la intencionalidad de las investigadoras en contribuir con objetivos de justicia social, en este caso, focalizando la atención en los ancianos (tanto los que tienen autonomía como los dependientes). Se trata de compensar posibles situaciones de privación, y de fortalecer lazos sociales mediante la cultura y la vivencia musical directa. 
En este proyecto, de acuerdo con los datos obtenidos mediante distintos instrumentos (entrevistas semiestructuradas con mayores, voluntarios y directivos, encuestas y registro de observaciones), se observa que este programa, desarrollado durante dos años, ha incidido fundamentalmente en la mejora de la calidad de vida de los dos grupos distintos de adultos mayores, lo cual coincide con los resultados obtenidos por investigaciones realizadas en otros contextos, que han puesto en evidencia que las actividades musicales activas influyen positivamente en los adultos mayores, mejorando la percepción subjetiva de bienestar y la interacción social (Coffman, 2002).

Con respecto a los voluntarios universitarios, aunque se trata de estudiantes de la Licenciatura en Artes Musicales, músicos prácticos cuyos planes de estudios están centrados en la adquisición del mayor nivel musical posible (técnico y expresivo), para su desarrollo personal y profesional futuro es importante que tomen conciencia y se formen para realizar aportaciones a la mejora de la sociedad, muy especialmente en el marco de una realidad donde las necesidades sociales muchas veces no son atendidas en forma completa por las entidades públicas, o bien están sujetas a los vaivenes políticos de los sucesivos gobiernos.

Para los estudiantes este voluntariado ha sido una oportunidad de enriquecimiento personal que promueve la actitud participativa en la mejora de la comunidad, y una experiencia de interpretación musical para un público diferente al de las salas tradicionales: es decir, han tenido la posibilidad de contribuir de manera activa y de tomar conciencia (ser responsables) de la mejora de los ancianos participantes, es decir, de contribuir al sentido social de la educación (Batlle, 2010). En las reuniones con el profesorado organizador, los estudiantes han planteado que la experiencia les ha hecho pensar en cuestiones totalmente diferentes a las puramente académicas y técnicas, tales como ofrecer un espacio musical reconfortante, o conversar de música con personas mayores que salen de su cotidianeidad gris gracias a estas audiciones.

Para los ancianos institucionalizados en hogares, y para aquéllos autoválidos que frecuentan los centros de jubilados de sus barrios, es una oportunidad de sentirse acompañados, de mejorar su estado general a través del contacto con los jóvenes y con la música, y de generar un espacio de reencuentro con un repertorio musical muchas veces conocido por ellos en etapas previas de su vida. Se trata de transformar estas instancias de estudio en un espacio socializador entre los adultos mayores de tal manera de ofrecerles una nueva perspectiva de vida, y de promover vínculos intergeneracionales constructivos para todas las partes.

Por otro lado, los ancianos institucionalizados presentan una serie de características, por una parte derivados de su edad y de su salud. La experiencia realizada mediante este programa pone de manifiesto que la música desempeña un papel importante en la mejora del estado general de estas personas, así como contribuye a la mejora de la reserva cognitiva y del estado anímico, lo que confirma los hallazgos de investigaciones previas (Thomson et al., 2005). Sin embargo, en la mayoría de los hogares de ancianos no se desarrollan actividades musicales, que tan beneficiosas serían para estas personas, muchas de ellas sin familia y sin contacto con el mundo exterior. Hay que tener en cuenta que en el contexto de la residencia de ancianas, el grupo que participó en este voluntariado estaba formado en su mayor parte eran melómanas. De acuerdo con lo que ellas manifestaron (tanto en las entrevistas semiestructuradas como a lo largo de las distintas sesiones) habían sido público habitual de conciertos de música clásica, y el 
hecho de volver a escuchar música en vivo ha supuesto para ellas un cierto retorno a la normalidad de su vida, del cual la residencia las había apartado. Por otra parte, la sociedad actual vive muchas veces ignorando a esta parte de nuestra comunidad, olvidando su responsabilidad ante la inclusión de personas ancianas: se ha comprobado que en las personas mayores puede contribuir al retardo del deterioro de las facultades físicas y mentales, propiciar la resocialización del anciano, proporcionar la recuperación psicológica y aumentar la autoestima; fomenta y desarrolla la comunicación, ayuda a conservar la estabilidad emocional, posibilita la autoexpresión y la integración social, desarrolla la creatividad, estimula la memoria aumentando la retención de información. De todo esto ha quedado testimonio en las respuestas que han dado tanto los voluntarios, como las personas ancianas, y los cuidadores y responsables de las residencias, al igual que las familias.

Finalmente, también es importante la labor realizada para lograr una toma de conciencia en los responsables de la Residencia, acerca de la necesidad de dotar de belleza y comunicación humana a las personas que están imposibilitadas de salir, de ir a escuchar un concierto, de conversar sobre música, y cómo todo esto mejora el estado de ánimo general, e ilumina la rutina cotidiana. Consideramos importante insistir que cuando los ancianos se encuentren institucionalizados se trate de organizar actividades musicales sistemáticas pues esto responde a la necesidad de mejorar su calidad de vida y bienestar.

\section{Agradecimientos}

Este trabajo se ha realizado gracias a la Convocatoria del Ministerio de Educación de Argentina para Proyectos de Voluntariado Universitario (2014 y 2015), cuyos proyectos se han desarrollado en 2015 y 2016.

\section{Referencias}

Batlle, R. (2010). Aprendizaje-servicio y entidades sociales. Aula de Innovación Educativa, 192, 6668.

Ciurana, M. y Cibanal, J. L. (2009). Aspectos psicoemocionales del pianista: Problemas y posibles soluciones. En M. Ciurana (Ed.), Los espacios pedagógicos del cuidado (pp. 52-59). Alicante: Consejo de Enfermería de la Comunidad Valenciana.

Coffman, D. (2002). Music and quality of life in older adults. Psychomusicology: A Journal of Research in Music Cognition, 18(2), 76-88. https://doi.org/10.1037/h0094050

De la Rubia, J. Sancho, P. y Cabanés, C. (2014). Impacto fisiológico de la musicoterapia en la depresión, ansiedad, y bienestar del paciente con demencia tipo Alzheimer. Valoración de la utilización de cuestionarios para cuantificarlo. European Journal of Investigation in Health, Psychology and Education, 4(2), 131-140.

De la Calle, C., García, J. y Giménez, P. (2007). La formación de la responsabilidad social en la universidad. Revista Complutense de Educación, 18(2), 47-66.

Di Virgilio, M., Marcos, M. y Mera, G. (2015). Las ciudades dentro de la ciudad: características sociodemográficas y habitacionales de la ciudad de Buenos Aires según sus tipos de hábitat. Revista Población de Buenos Aires, 12, 33-57.

European Comission. (2012). The 2012 ageing report. Economic and budgetary projections for the EU27 Member States. Bruselas: Secretariat of the Economic Policy Committee. 
Kandel, V. (2013). Hay algunos que sienten que esto les parte la cabeza: Notas sobre experiencias de aprendizaje-servicio en la Universidad de Buenos Aires. Revista Internacional de Educación para la Justicia Social (RIEJS), 2(2), 177-193.

Lehmberg, L. y Fung, V. C. (2010). Benefits of music participation for senior citizens: A review of the literature. Music Education Research International, 4, 19-30.

Loreto, V. y Matallanes, B. (2012). Desarrollo y evaluación de competencias psicosociales en estudiantes universitarios a través de un programa de aprendizaje-servicio. Revista Internacional de Educación para la Justicia Social (RIEJS), 2(2), 155-176.

Mayán, J. M., Rodríguez de Vera, B., Núñez, M. J., Freire-Garabal, M., Rodríguez Martínez, A. y Seijas, B. (2001). Musicoterapia: Técnica psicomotriz gerontológica. Santiago de Compostela: Sega Ediciones.

Thomson, R. G., Moulin, C. J. A., Hayre, S. y Jones, R. W. (2005). Music enhances category fluency in healthy older adults and alzheimer's disease patients. Experimental Aging Research, 31(1), 91-99. https://doi.org/10.1080/03610730590882819

\section{Breve CV de las autoras}

\section{Susana Sarfson}

Doctora en Filosofía y Ciencias de la Educación. Profesora Superior de Piano. Profesora Superior de Clave. Licenciada en Filología Hispánica. Es Profesora Titular de Universidad en el área de Didáctica de la Expresión Musical (Universidad de Zaragoza) con docencia de grado, máster y doctorado. Investigadora principal en proyectos competitivos desarrollados en España e Hispanoamérica. Directora de Tesis Doctorales. Ha presentado numerosos trabajos de investigación en congresos nacionales e internacionales. Autora de publicaciones de investigación (artículos y libros). Sexenio vivo CNEAI. Es miembro del comité científico de las Revistas Educare (Costa Rica), Revista Complutense de Investigación en Educación Musical (España), y del Boletín de Investigación en Educación Musical (ADOMU, Argentina). ORCID ID: 0000-00020785-4952. Email: sarfson@unizar.es

\section{María Claudia Albini}

Profesora Superior de Música; profesora Superior de Inglés; licenciada en Educación; licenciada en Artes Musicales; doctoranda en Artes. En Argentina es docente de nivel secundario, terciario y universitario. Investigadora universitaria categorizada. Asesora del Instituto de Investigación en Artes Musicales-DAMus-UNA. Miembro del Banco de Evaluadores de la Red Nacional de Extensión Universitaria (REXUNI). Directora de tesinas de grado. Expositora en numerosas reuniones científicas. Evaluadora acreditada de la Universidad de Cambridge (Cambridge ESOL examinations). Representante en Argentina de la International Child Art Foundation (Washington). Editora del ARTESOL ESP journal y referato del ARTESOL EFL journal. Directora del proyecto de Voluntariado Universitario "En modo mayor-Música para nuestros abuelos". ORCID ID: 0000-0003-2027-2209. Email: mariaclaudia.albini@gmail.com 